Meta

Journal des tradlucteurs

Translators' Journal

\title{
Vocabulaire relatif aux personnes âgées, à la retraite et au vieillissement
}

\section{Journal officiel de la République Française}

Volume 31, numéro 4, décembre 1986

URI : https://id.erudit.org/iderudit/003593ar

DOI : https://doi.org/10.7202/003593ar

Aller au sommaire du numéro

Éditeur(s)

Les Presses de l'Université de Montréal

ISSN

0026-0452 (imprimé)

1492-1421 (numérique)

Découvrir la revue

Citer ce document

Journal officiel de la République Française (1986). Vocabulaire relatif aux personnes âgées, à la retraite et au vieillissement. Meta, 31(4), 397-403.

https://doi.org/10.7202/003593ar d'utilisation que vous pouvez consulter en ligne. 


\section{VOCABULAIRE RELATIF AUX PERSONNES ÂGÉES,} À LA RETRAITE ET AU VIEILLISSEMENT

Arrêté du 13 mars 1985 relatif à l'enrichissement du vocabulaire relatif aux personnes âgées, à la retraite et au vieillissement du ministère des Affaires sociales et de la Solidarité nationale de la République française.

LISTE No 1

Les expressions et termes figurant dans cette liste sont approuvés.

âgisme, n.m.

Domaine : Vie sociale

Définition : Attitude ou comportement visant à déprécier les individus du fait de leur âge.

Note : Ce terme est formé par analogie avec racisme. Il s'emploie plus particulièrement pour désigner la discrimination dont sont victimes les *personnes âgées.

Voir aussi : Discrimination sociale, inégalité sociale, ségrégation sociale.

appartement thérapeutique, n.m.

Domaine : Action sociale - Santé publique

Définition : Dans le cadre des *alternatives à l'hospitalisation, structure d'hébergement conçue pour un petit groupe de personnes malades ou *handicapées dont le *maintien à domicile n'est plus possible ou souhaitable.

Note : Les appartements thérapeutiques ont été utilisés d'abord pour des malades mentaux, avant d'être employés pour des *handicapés et des *personnes âgées. 


\section{centre de jour, n.m.}

Domaine : Santé publique — Gérontologie — Personnes âgées

Définition : Centre où des *personnes âgées peuvent être accueillies et soignées pendant la journée.

Note : C'est une unité de soins installée dans un quartier, qui assure une prise en charge globale de la * personne âgée atteinte d'une affection chronique ou d'un *handicap physique ou mental. Le centre est destiné à éviter l'hospitalisation ou à en réduire la durée. Il a donc un rôle d'information, de prévention et de soins.

Voir aussi : alternative à l'hospitalisation, hôpital de jour.

club de personnes âgées, n.m.

Domaine : Vie sociale - Gérontologie — Personnes âgées

Synonyme : club de retraités, n.m.

club du $3^{e}$ âge, n.m.

Définition : Groupe de *personnes âgées se réunissant pour partager des activités non lucratives : jeux de société, sports, activités culturelles, voyages, etc.

Note : Les clubs sont quelquefois affiliés à une fédération départementale, régionale, nationale ou internationale. Ils peuvent relever d'un *centre d'action sociale.

\section{coordonnateur, n.m.}

\section{coordonnatrice, n.f.}

Domaine : Action sociale - Gérontologie - Personnes âgées

Définition : À l'intérieur d'un département, personne chargée d'assurer la coordination des actions menées par les services, organismes et institutions en faveur des retraités et personnes âgées dans une circonscription géographique de taille variable.

Voir aussi : sectorisations

cohabitation des générations, n.f.

Domaine : Vie sociale

Définition : Situation dans laquelle habitent ensemble des membres d'une même famille, appartenant à des * générations différentes.

Note : Le terme s'emploie surtout pour désigner la cohabitation des parents âgés et de leurs enfants majeurs. Voir aussi : décohabitation

\section{décohabitation, n.f.}

Domaine : Vie sociale - Gérontologie - Personnes âgées

Définition : 1. Passage d'une situation de *cohabitation des générations à une séparation de domicile entre des parents âgés et leurs enfants majeurs.

2. Par extention diminution au sein d'une population du taux de *cohabitation des générations.

\section{degré de vieillissement, n.m.}

Domaine : Démographie - Gérontologie - Vieillissement

Définition : Proportion des *personnes âgées dans une population.

Voir aussi : gérité

\section{dépendance, $n . f$.}

Domaine : Médecine

Définition : Situation d'une personne qui en raison d'un déficit anatomique ou d'un trouble physiologique ne peut remplir des fonctions, effectuer des gestes essentiels à la vie quotidienne sans le concours d'autres personnes ou le recours à une prothèse, un remède, etc. 
Note : La dépendance du malade, de l'invalide, du vieillard n'aboutit le plus souvent à la privation ou à l'abdication de son autonomie que par le cumul et la combinaison de dépendances ajoutées, qui sont d'un autre ordre que la pathologie organique ou fonctionnelle, par exemple d'ordre économique, financier, social, etc.

Voir aussi : perte d'autonomie

foyer pour personnes âgées, n.m.

Domaine : Vie sociale

Définition : Lieu où les personnes âgées peuvent se rencontrer.

foyer-restaurant, n.m.

Domaine : Action sociale - Gérontologie - Personnes âgées

Définition : Établissement dans lequel les *personnes âgées peuvent prendre le repas de midi, et d'où elles peuvent emporter le repas du soir.

Note : La participation financière de la *personne âgée dépend de ses ressources. Les repas peuvent éventuellement être portés à domicile pour les personnes qui sont dans l'impossibilité de se déplacer. Le foyerrestaurant peut être géré par un centre d'action sociale ou une association privée.

Voir aussi : service de repas à domicile

foyer-soleil, n.m.

Domaine : Action sociale - Gérontologie - Personnes âgées

Définition : Ensemble de logements proches les uns des autres et disposant en commun de services collectifs.

Note : Le foyer-soleil peut résulter de l'extension d'un *logement-foyer ou s'intégrer dans des immeubles d'habitation où des logements sont réservés aux *personnes âgées.

gériatre, n.m. ou n.f.

Domaine : Médecine - Gérontologie - Vieillissement

Définition : Médecin spécialiste qui se consacre à la *gériatrie.

gériatrie, n.f.

Domaine : Médecine - Gérontologie - Vieillissement

Définition : Discipline médicale consacrée à l'étude de la santé et aux soins des * personnes âgées.

Voir aussi : gérontologie

gérontologie, n.f.

Domaine : Sciences sociale - Gérontologie

Définition : Étude de la vieillesse, de la population âgée et de tous les aspects du vieillissement.

Voir aussi : gériatrie

gérontologue, n.m. ou n.f.

Domaine : Sciences sociales - Gérontologie

Définition : Personne qui se consacre à la *gérontologie.

hébergement collectif, n.m.

Domaine : Action sociale - Gérontologie - Retraite

Définition : Substitut de domicile qui permet à la *personne âgée de bénéficier de services collectifs.

Note : Exemples : *logement-foyer, structure d'*hébergement temporaire, *maison de retraite. 


\section{hébergement temporaire, n.m.}

Domaine : Action sociale - Gérontologie - Retraite

Définition : Accueil, limité dans le temps, dans des structures d'habitation autres que leur domicile, de *personnes âgées dont la situation est provisoirement précaire.

Note : Ce mode d'hébergement vise à éviter l'hospitalisation temporaire ou définitive des *personnes âgées, ou encore leur placement dans unè institution spécialisée. À ce titre, il s'inscrit dans la politique de maintien à domicile.

Voir aussi : alternative à l'hospitalisation, appartement d'accueil, résidence d'hébergement temporaire.

\section{hospice, n.m.}

Domaine : Action sociale - Gérontologie — Personnes âgées

Définition : Traditionnellement, maison d'assistance où l'on reçoit les orphelins, les incurables de tous âges, les infirmes et les vieillards leur assurant, le cas échéant, les soins nécessaires.

Note : Depuis 1975, les hospices doivent être transformés en *maison de retraite avec éventuellement une *section de cure, ou en établissement hospitalier de long séjour.

Voir aussi : unité de long séjour

\section{logement-foyer, n.m.}

Domaine : Action sociale — Gérontologie - Personnes âgées

Définition : Groupe de logements ou de chambres autonomes assortis de services collectifs dont l'usage est facultatif.

Note : Ces logements-foyers sont destinés aux *personnes âgées capables de vivre de manière habituelle dans un logement indépendant, mais ayant occasionnellement besoin d'être aidées.

Le terme foyer-logement ne doit pas être utilisé.

Voir aussi : foyer-soleil

\section{maison d'accueil pour personnes âgées, n.f.}

Abréviation : M.A.P.A., n.f.

Domaine : Action sociale — Gérontologie - Personnes âgées

Définition : Établissement d'hébergement social conçu pour des *personnes âgées ayant perdu leur *autonomie.

Voir aussi : maison de retraite, unité de séjour.

maison de retraite, $n$,f.

Domaine : Action sociale - Gérontologie - Retraite

Définition : Établissement d'hébergement social, offrant une prise en charge globale de la *personne âgée par l'hébergement, la restauration, les services essentiels de la vie courante, l'animation à caractère culturel, etc.

Note: 1. Ces établissements peuvent être classés en maisons de retraite publiques ou privées conventionnées, avec prix de journée fixé par la direction départementale de l'action sanitaire et sociale, et en maisons de retraite privées non conventionnées dans lesquelles le prix demandé est libre.

2. La *personne âgée hébergée doit régler un prix de journée que l'aide sociale peut dans certains cas prendre en charge.

migration de retraité, n.f.

Domaine : Démographie - Gérontologie - Retraite

Définition : Déplacement d'une personne transférant sa résidence à l'occasion de la retraite.

Voir aussi : immigration de retraités, migrant âgé. 
personne âgée, n.f.

Domaine : Vie sociale - Gérontologie — Personnes âgées

Définition : Personne plus âgée que la moyenne des autres personnes de la population dans laquelle elle vit.

Note : 1. Dans l'opinion courante, ce concept sous-entend souvent que cette personne n'a plus d'activité rémunérée et qu'elle a des capacités diminuées.

2. Il est parfois employé de manière imprécise et inadéquate.

3. L'expression personne âgée est cependant commode pour remplacer celles de vieux, vieilles, vieillards car le mot vieux a souvent des connotations négatives de déclin, de déchéance, d'obsolescence ou d'incapacité ; on peut être âgé, au sens défini plus haut, sans être vieux en ce sens-là.

4. Plus récemment on associe les personnes âgées et les retraités parce que en France, en ce dernier quart du $\mathrm{xX}^{\mathrm{c}}$ siècle, on peut être encore vieux ou âgé sans être retraité, et plus souvent on peut être retraité bien avant d'être vieux au sens de diminué.

\section{perte d'autonomie, $n . f$.}

Domaine : Médecine - Sciences sociales

Définition : Impossibilité pour une personne d'effectuer certains actes de la vie courante, dans son environnement habituel.

Note : Les personnes ayant perdu certaines de leurs capacités sont parfois dites semi-autonomes.

Voir aussi : autonomie, dépendance, handicap.

plan gérontologique départemental, n.m.

Domaine : Action sociale - Gérontologie

Définition : Plan descriptif des différents établissements et services prenant en charge des *personnes âgées dans un département.

\section{préretraite, n.f.}

Domaine : Action sociale - Gérontologie - Retraite

Définition : 1. Expression usuelle et impropre qui désigne certaines *allocations versées jusqu’à la date normale de son départ en retraite à un salarié de cinquante-cinq ans ou plus ayant cessé de travailler

2. Situation dans laquelle se trouve un salarié qui reçoit l'une de ces allocations.

Note : Exemples d'allocations de préretraite : préretraite d'entreprise, garantie de ressources, allocation versée dans le cadre de fonds national de l'emploi ou des contrats de solidarité.

Voir aussi : retraite anticipée

\section{régime de retraite, n.m.}

Domaine : Action sociale - Gérontologie - Retraite

Définition : 1. Ensemble des avantages sociaux servis au titre de l'assurance-vieillesse par un organisme légal ou conventionnel de sécurité sociale.

2. Organisme légal ou conventionnel de sécurité sociale servant des pensions de vieillesse.

Voir aussi : caisse de retraite

\section{résidence d'hébergement temporaire, n.f.}

Domaine : Action sociale - Gérontologie - Personnes âgées

Définition : Établissement d'hébergement, médicalisé ou non, visant à assurer la sécurité matérielle, affective et psychologique des *personnes âgées pour une durée de séjour qui peut varier de quelques jours à quelques semaines.

Voir aussi : alternative à l'hospitalisation, appartement d'accueil, hébergement temporaire, maintien à domicile. 
résidence pour personnes âgées, n.f.

Domaine : Action sociale

Définition : Logement d'habitation conçu pour les *personnes âgées.

Voir aussi : logement-foyer, maison de retraite.

retraite, n.f.

Domaine : Vie sociale - Gérontologie - Retraite

Définition : 1. Situation dans laquelle se trouve une personne qui s'est retirée de la vie professionnelle, soit volontairement, soit après avoir atteint une limite d'âge.

2. Cessation d'activité professionnelle à un certain âge.

3. Par extension, pension que perçoit un retraité.

section de cure médicale, n.f.

Domaine : Action sociale - Gérontologie — Personnes âgées

Définition : Modalité de prise en charge par un régime de protection sociale suivant laquelle un établissement pour * personnes âgées est autorisé à organiser des soins de caractère médical pour une partie des personnes hébergées.

Note : Le mot section peut sembler improprement utilisé, car il n'implique pas que les personnes ainsi prises en charge soient séparées des autres. Le nombre de lits de cure médicale composant cette section est fixé annuellement par convention.

services à domicile, n.m.pl.

Domaine : Action sociale - Gérontologie - Personnes âgées

Définition : *Prestations telles que aide ménagère, soins, repas à domicile, petits travaux, destinés à aider, là où elles habitent, des *personnes âgées dont lautonomie de vie est restreinte.

soutien à domicile, n.m.

Domaine : Action sociale - Gérontologie — Personnes âgées

Définition : Ensemble d'aides matérielles financières et psychologiques qui contribuent à améliorer les conditions de vie d'une * personne âgée, handicapée ou malade, dans son cadre habituel de vie.

Voir aussi : maintien à domicile

unité de long séjour, n.f.

Domaine : Action sociale - Santé publique

Définition : Établissement ou partie d'établissement qui a pour mission d'assurer l'hébergement de longue durée des personnes ayant perdu leur autonomie de vie et dont l'état nécessite une surveillance médicale constante et des traitements d'entretien.

Voir aussi : hospice, unité de moyen séjour.

unité de moyen séjour, n.f.

Abréviation : Moyen séjour, n.m.

Domaine : Action sociale - Santé publique

Définition : Service hospitalier permettant d'assurer, après la phase aiguë de la maladie, le prolongement temporaire de soins actifs ainsi que les traitements nécessaires à la réadaptation en vue du retour à une existence autonome.

Voir aussi : unité de long séjour 


\section{vieillissement, n.m.}

Domaine : Médecine - Sciences sociales - Gérontologie - Vieillissement

Définition : 1. Transformation avec l'avance en âge de tout organisme vivant.

2. Par extension, accroissement de la proportion de *personnes âgées dans un groupe social ou une population.

Note : Si certaines modalités de ce processus sont réversibles, l'ensemble est ou paraît être irréversible. Ce processus résulte d'un jeu complexe de progrès, comme la croissance en taille, de régressions, comme la diminution de l'acuité visuelle, de compensations, qui varient d'un individu à un autre.

\section{LISTE No 2}

L'emploi des expressions et termes figurant dans cette liste est recommandé.

\section{gérescence, n.f.}

Domaine : Démographie — Gérontologie — Vieillissement

Définition : Processus de vieillissement d'une population.

Note : Ce néologisme a été proposé pour éviter toute confusion avec le *vieillissement de l'individu.

Voir aussi : gérité, vieillissement démographique.

\section{gérité, n.f.}

Domaine : Démographie

Définition : État d'une population vieillie.

Note : Ce néologisme a été proposé pour éviter la confusion avec le vieillissement de l'individu.

Voir aussi : gérescence, vieillissement démographique.

\section{nursage, n.m.}

Domaine : Médecine - Soins infirmiers

Définition : Ensemble des soins infirmiers destinés à éviter ou à limiter les complications que risque d'entraîner une maladie grave invalidante ou prolongée : soins d'hygiène corporelle, prévention des escarres, surveillance de l'alimentation, etc.

Note : Le terme nursing ne doit pas être employé en français.

\section{retraitologie, n.f.}

Domaine : Sciences sociales

Définition : Étude de la retraite dans tous ses aspects, notamment juridiques, financiers, économiques, sociaux ou psychologiques. 